\title{
Quality of Merge-Refactorings for Product Lines
}

\author{
Julia Rubin ${ }^{1,2}$ and Marsha Chechik ${ }^{1}$ \\ ${ }^{1}$ University of Toronto, Canada \\ 2 IBM Research in Haifa, Israel \\ mjulia@il.ibm.com, chechik@cs.toronto.edu
}

\begin{abstract}
In this paper, we consider the problem of refactoring related software products specified in UML into annotative product line representations. Our approach relies on identifying commonalities and variabilities in existing products and further merging those into product line representations which reduce duplications and facilitate reuse. Varying merge strategies can lead to producing several semantically correct, yet syntactically different refactoring results. Depending on the goal of the refactoring, one result can be preferred to another. We thus propose to capture the goal using a syntactic quality function and use that function to guide the merge strategy. We define and implement a quality-based mergerefactoring framework for UML models containing class and statechart diagrams and report on our experience applying it on three case-studies.
\end{abstract}

\section{Introduction}

A software product line $(S P L)$ is a set of software-intensive products sharing a common, managed set of features that satisfy the specific needs of a particular market segment [4]. SPL engineering practices capitalize on identifying and managing commonalities and variabilities across the whole product portfolio and promote systematic software reuse. SPL commonalities represent artifacts that are part of each product of the product line, while SPL variabilities - those specific to some but not all products. Benefits of applying SPL engineering practices include improved time-to-market and quality, reduced portfolio size, engineering costs and more [4]9]. Numerous works, e.g., [9], promote the use of SPL practices for model-based development of complex embedded systems. Often, variants of such systems are developed for different customers and are represented and implemented using visual structural and behavioral models.

In reality, however, SPLs often emerge ad-hoc, when companies have to release a new product that is similar, yet not identical, to existing ones. Under tight project scheduling constraints, development teams resort to copying artifacts from one of the existing products and later modifying them independently from the original version [15 18] (the clone-and-own approach).

Cloned artifacts require synchronization: changes in one artifact might need to be repeated for all variants. In addition, it is difficult to propagate a new feature implemented in one variant into another or to define a new product by selectively "picking" some, but not all, features from the existing variants. As the result, when product variants are realized via cloning, development and maintenance efforts increase as the number of products grows. To deal with the complexity of SPL development, some approaches,

V. Cortellessa and D. Varró (Eds.): FASE 2013, LNCS 7793, pp. 83-98, 2013.

(C) Springer-Verlag Berlin Heidelberg 2013 
e.g., [2], advocate refactoring legacy cloned products into "single-copy" representations, eliminating duplications and explicating variabilities.

Numerous works provide guidelines and methodologies for building product line representations out of legacy systems, e.g., [14]8]. Most of such approaches, however, involve a manual review of code, design and documentation of the system aiming at identifying the set of product line features, as well as the set of components which implement these features. This manual step is time-consuming, and, in many cases, impedes adoption of SPL techniques by organizations.

Automated approaches for mining legacy product lines and refactoring them into feature-oriented product line representations have also been proposed [7 18 | 30 [25]. In our earlier work [22 24], we focused on refactoring model-level cloned product variants and proposed a configurable merge-refactoring algorithm, merge-in, applicable to refactoring models of different types (e.g., UML, EMF and Matlab/Simulink). Our algorithm identifies similar and different elements of the input models using parameterizable compare and match operators, and then constructs a refactored model using a merge operator. The resulting product line model contains reusable elements representing corresponding merged elements of the original models. In [24], we formally proved that merge-in produces semantically correct refactorings for any set of input models and parameters: a refactored model can derive exactly the set of original products, regardless of particular parameters chosen and implementations of compare / match / merge used, if they satisfy well-defined correctness properties (e.g., "each element produced by merge originates from an element of at least one input model”).

Varying merge-in parameters allows producing different syntactic representations of the resulting product line due to different possible ways to match input model elements. All these representations are semantically equivalent and derive the same set of products. However, not all possible syntactic representations are desirable. Moreover, depending on the goal of the refactoring, one representation might be preferable to another. For example, a goal of the refactoring can be to highlight the variability points between the products, eliminating the "unnecessary" variability and creating a more homogeneous product portfolio. Another can be to maximize the comprehensibility of the resulting model by minimizing variability annotations for elements of a certain type. Yet another can be to reduce the size of the resulting refactoring - this might happen if the models are used for execution or code generation rather than human inspection. These different goals induce different product line representations.

Example. Consider the UML model fragments in Fig.1 (a,b) depicting two representative parts of real-life products developed by an industrial partner (since partner-specific details are confidential, we move the problem into a familiar domain of washing machines). Fig. 1(a) shows the Controller, Washer and Dryer classes of a washing machine, together with snippets of Controller's and Dryer's behaviors specified by UML statechart models. The wtrLevel attribute of Controller is used to specify the desired water level. When the water is filled to that level and heated to $30^{\circ} \mathrm{C}$, the washing machine controller notifies Washer that it can start operating and transitions from the Locking to the Washing state. After finishing washing, the controller initiates Dryer and transitions to the Drying state. Dryer operates for 45 minutes and returns the control to the Controller's statechart (by sending an appropriate signal which is 


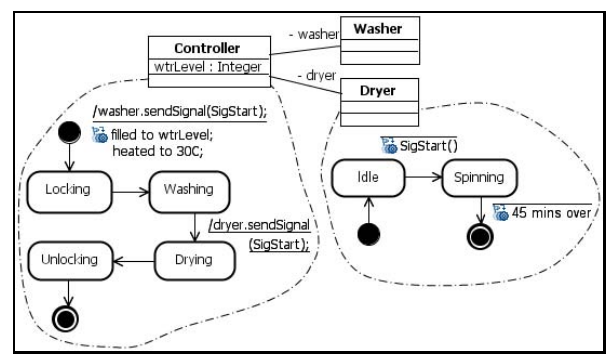

(a) An Original Model with Dryer.

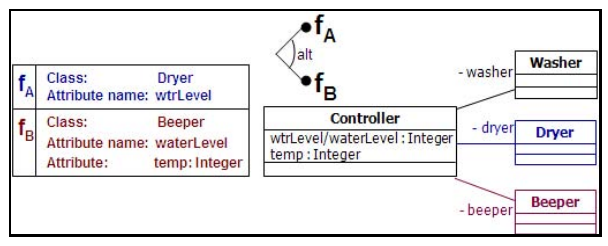

(c) Refactoring \#1.

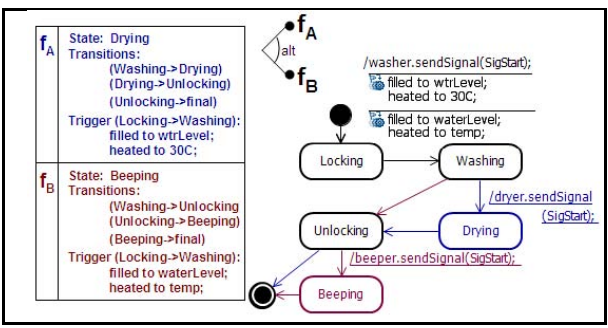

(e) Controller statechart Refactoring \#1.
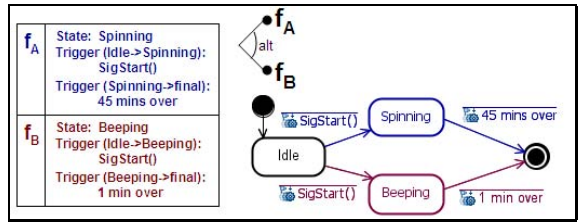

(g) Dryer and Beeper Refactoring \#1.

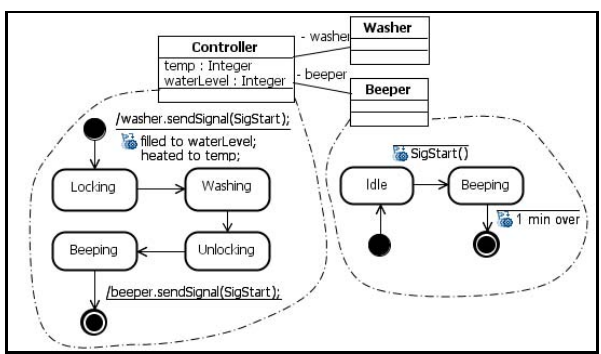

(b) An Original Model with Beeper.

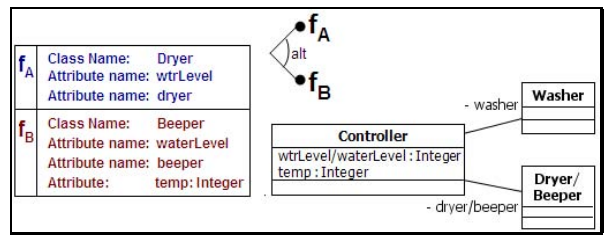

(d) Refactoring \#2.

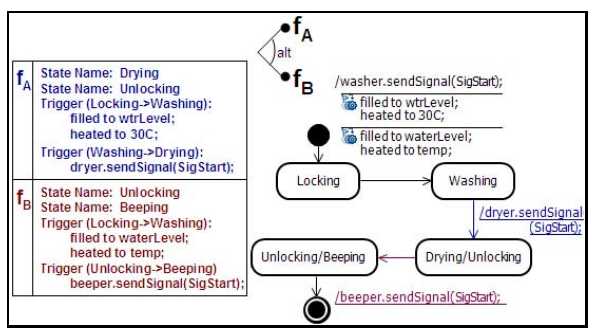

(f) Controller statechart Refactoring \#2.

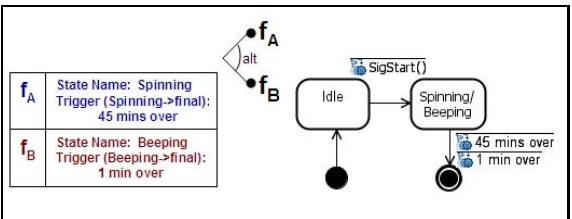

(h) Dryer and Beeper Refactoring \#2.

Fig. 1. Fragments of Washing Machine models and some of their refactorings

omitted from the picture to save space). Then, the washing machine is unlocked, and the wash cycle stops. Fig. 1(b) shows a similar washing machine model which lacks the dryer but has a beeping function indicating the end of the wash cycle by signalling for 1 minute. In addition, in this model, the temp and waterLevel attributes control the desired water temperature and water level, respectively.

These two products have a large degree of similarity and can be refactored into annotative SPL representations, where duplications are eliminated and variabilities are explicated. We consider only those refactorings that preserve the behavior of existing products rather than allowing novel feature combinations (e.g., a product with both the dryer and the beeper). Even with this simplification, several choices emerge. For 
example, the two input models in Fig. 1 1 a, b) can be combined as shown in Fig. 1(c) where the Controller classes of both input models are matched and merged together, while the Dryer and the Beeper classes are unmatched and thus both copied to the result "as is", together with their corresponding statecharts. Another choice is shown in Fig. 1(d) where these two classes are matched and merged together, producing either a representation in Fig. 1 $\mathrm{g}$ ) or in (h). Combining statecharts of Controller classes can also result in two possible representations, as shown in Fig. 1(e) and (f). That is, there are six possible refactoring options: Fig 1 $(\mathrm{c}, \mathrm{e}),(\mathrm{c}, \mathrm{f}),(\mathrm{d}, \mathrm{e}, \mathrm{g}),(\mathrm{d}, \mathrm{e}, \mathrm{h}),(\mathrm{d}, \mathrm{f}, \mathrm{g})$ and $(\mathrm{d}, \mathrm{f}, \mathrm{h})$.

In each of the cases, the created models are controlled by a set of features, depicted in the middle upper part of each figure. Since the refactored product line in our example encapsulates only the original input products, we have just two alternative features representing these products $-f_{A}$ and $f_{B}$. Product line model elements are annotated by these features, as shown in a table on the left-hand side of each figure. The set of annotations specifies elements to be selected given a particular feature selection: selecting $f_{A}$ filters out all elements annotated with $f_{B}$, which derives the original input model shown in Fig. 1(a) from each of the refactorings. Likewise, selecting feature $f_{B}$ derives the original model shown in Fig. 1(b) For a refactoring that aims at maximizing the comprehensibility of the resulting model, the best representation is the one shown in Fig. 1] (c, e) since it has the least number of classes and states with variable names and the least number of variable statecharts. However, for a refactoring that aims at reducing the size of the result, the best representation is the one in Fig. 1 $(\mathrm{d}, \mathrm{f}, \mathrm{h})$, as it contains three classes and six states only, compared to the refactoring in Fig. 11 c, e) which has four classes and nine states.

Contributions. We consider the problem of integrating several distinct products specified in UML into an annotative product line representation using merge-refactorings. (1) We argue that there can be multiple syntactically different product line models that represent the same set of products. All such models are valid, but not all are desired. Explicating the goal of the refactoring can help produce those that better fit the user intention. (2) We propose to capture the goal of the refactoring using a quantitative quality function, comprised of a set of measurable syntactic metrics. This function is used to evaluate the produced refactorings and to guide the merge-refactorings process towards a desired result. (3) We present an approach for exploring the set of different refactorings with the goal of identifying the one that maximizes the value of a given quality function. (4) We report on an implementation of a quality-based merge-refactoring framework for UML models containing class and statechart diagrams, which realizes the merge-in algorithm introduced in our earlier work [24]. We use the implemented framework for evaluating the effectiveness of our approach using several example product lines specified in UML, including one contributed by an industrial partner.

The remainder of this paper is organized as follows. The details on annotative product line representations and the merge-in refactoring algorithm are given in Sec. 2 Our quality-based merge-refactoring framework is described in Sec. 3. In Sec.4, we present an implementation of the framework. We describe our experience applying it to three case studies in Sec.5, A discussion and a description of related work are given in Sec.6. Sec.7 concludes the paper and outlines future research directions. 


\section{Refactoring Software Product Lines}

In this section, we describe software product line models annotated by features. We also give the necessary background on model merging, used as a foundation of our merge-in product line refactoring algorithm, and summarize the merge-in algorithm itself.

Software Product Lines. SPL approaches can largely be divided into two types: compositional which implement product features as distinct fragments and allow generating specific products by composing a set of fragments, and annotative which assume that there is one "maximal" representation in which annotations indicate the product features that a particular fragment realizes [11]3]. A specific product is obtained by removing fragments corresponding to discarded features. Similarly to [3], our experience is that the annotative approach, which reminds code-level \#ifdef statements, is easier to adopt in practice, as it does not require a paradigm shift in the way software is being commonly developed, especially in the embedded domain. We thus follow this approach here.

A feature model is a set of elements that describe product line features and a propositional formula defined over these features to describe relationships between them. A feature configuration, defining a product of a product line, is a sub-set of features from the feature model that respect the given relationships. An annotative product line is a triple consisting of a feature model, a domain model (e.g., a set of UML classes and statecharts), and a set of relationships that annotate elements of the domain model by the features of the feature model. Fig. 1(c-h) present snippets of domain models (righthand side of each figure) whose elements are connected to features from a feature model (top-middle part of each figure) using annotation relationships (left-hand side of each figure). In this case, features $f_{A}$ and $f_{B}$ are alternative to each other, i.e., the propositional formula that specifies their relationship is $\left(f_{A} \vee f_{B}\right) \wedge \neg\left(f_{A} \wedge f_{B}\right)$. Thus, the only two valid feature configurations are $\left\{f_{A}\right\}$ and $\left\{f_{B}\right\}$.

A specific product derived from a product line under a particular configuration is the set of elements annotated by features from this configuration. For example, the class diagrams in Fig. 1(a) and Fig. 1(b) can be derived from the product line in Fig. 1(d) under the configurations $\left\{f_{A}\right\}$ and $\left\{f_{B}\right\}$, respectively.

Model Merging. Model merging consists of three steps: compare, match and merge. Compare is a heuristic function that calculates the similarity degree, a number between 0 and 1, for each pair of input elements. It receives models $M_{1}, M_{2}$ and a set of empirically assigned weights which represent the contribution of model sub-elements to the overall similarity of their owning elements. For example, a similarity degree between two classes is calculated as a weighted sum of the similarity degrees of their names, attributes, operations, etc. Comparing Washer classes in Fig.11(a, b) to each other yields 1, as these classes are identical in the presented model fragment. Comparing Controller classes yields a lower number, e.g., 0.8, as the classes have different owned properties and behaviors.

Match is a heuristic function that receives pairs of elements together with their similarity degree and returns those pairs of model elements that are considered similar. Match uses empirically assigned similarity thresholds to decide such similarity. Consider the above example, where Washing classes had a calculated similarity degree of 1 
and Controller classes had a similarity degree of 0.8 : setting class similarity threshold to 0.75 results in matching both pairs of classes, while setting it to 0.85 results in matching only the Washing classes.

Finally, merge is a function that receives two models together with pairs of their matched elements and returns a merged model that contains all elements of the input, such that matched elements are unified and present in the result only once. For example, Controller classes in Fig. 1 $(a, b)$ can be unified as shown on the right-hand side of Fig. 1(e): matched states Locking, Washing and Unlocking are unified, while unmatched states Drying and Beeping are just copied to the result together with their corresponding transitions. While the compare and match functions rely on heuristically determined weights and similarity degrees, merge is not heuristic: its output is uniquely defined by the input set of matched elements.

Merging-in Product Lines. We now describe the merge-in refactoring algorithm [24] that puts together input products into an annotative product-line representation. Constructing an annotative product line model consists of three steps: creating a domain model, creating a feature model, and specifying annotation relationships between the features and the domain model elements. For creation of a domain model, merge-in relies on model merging, described above. Feature models are created using an approach where features represent the original input products and are defined as alternatives to each other, so only the original products can be derived from the constructed product line model. Domain model elements are annotated by these features according to the product(s) that contributed them. For the example in Fig. 1(e), state Drying is annotated by feature $f_{A}$ while state Beeping is annotated by $f_{B}$. State Washing is common (it exists in both input models) and thus is annotated by both features. Annotations of common elements are not shown in the figure to save space.

Any input product $M$ can be seen as a "primitive" product line with only one feature $f_{M}$, one feature configuration $\left\{f_{M}\right\}$, and a set of annotations that relate all model elements to that feature. This representation can derive exactly one product $-M$. Thus, the most generic form of the merge-in operator obtains as input two (already constructed) product lines, each of which can be a "primitive" product line representing one input model. For example, when combining the two products in Fig. 1 a, b), we implicitly convert each of them into a product line and then merge-in them together. One possible outcome of that process is shown in Fig. 1 ( c, e), where the features representing the original models are denoted by $f_{A}$ and $f_{B}$ and defined as alternatives. In this case, Dryer and Beeper classes are unmatched.

Varying compare and match parameters, as well as varying the order in which input models are combined, defines the exact shape of the refactoring outcome. Two products in Fig. 1 (a, b) can also be combined as shown in Fig. 1(d, f), where a lower class similarity threshold results in Dryer and Beeper classes being matched and merged.

All possible refactorings constructed by the algorithm are semantically "correct", each deriving the exact set of input models, regardless of the parameters chosen and regardless of the order in which input products are merged-in. The correctness of the merge-in operator relies on "reasonable" behavior of model compare, match and merge algorithms. Formal correctness properties of those algorithms are specified in [24]. 


\section{Quality-Based Merge-Refactoring Framework}

Even though all refactorings produced by the merge-in algorithm are semantically equivalent and correct, not all refactorings are desirable: depending on the goal of the refactoring, one representation can be preferred to another. The main objectives of our quality-based product line merge-refactoring framework are thus to (1) allow the user to explicate the goal of the refactoring process and (2) drive the refactoring process towards the result that best fits the user intention. We depict our approach in Fig. 2 and describe it below.

\subsection{Explicating the Refactoring Goal}

We explicitly capture the goal of the refactoring using a quantitative quality function. Since in our work we focus on syntactic properties of mergerefactorings, the quality function is built from a set of metrics - syntactically measurable indicators representing specific refactoring objectives (see Fig. 2). Typ-

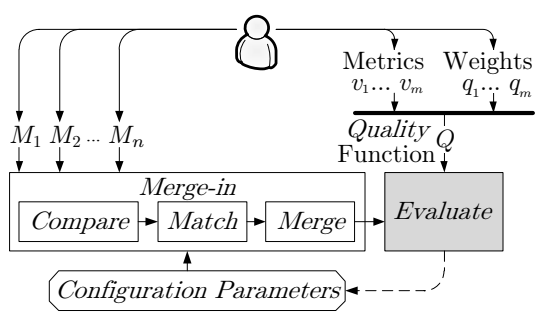

Fig. 2. Merge-Refactoring Framework ically, such metrics can assess the size of the resulting model, determine the degree of object coupling, cohesion in methods, weighted number of methods per class and more [17]. The metrics can be reused across different organizations and domains. Each concrete quality function $Q$ assigns weights $q_{1} \ldots q_{m}$ to different metrics $v_{1} \ldots v_{m}$, indicating their "importance" in the context of a specific application domain and allows the user to specify the desired outcome of the refactoring in an explicit manner.

More formally, given a product line model $\mathcal{P} \mathcal{L}$, the quality function returns a number between 0 and 1 , representing $\mathcal{P} \mathcal{L}$ 's "quality":

$$
\operatorname{quality}(\mathcal{P} \mathcal{L}, \mathbb{V}, \mathbb{Q})=\sum_{i=1 \ldots n} q_{i} * v_{i}(\mathcal{P} \mathcal{L}),
$$

where $\mathbb{V}=v_{1}, \ldots, v_{n}$ is a set of measurable metrics that, given $\mathcal{P} \mathcal{L}$, produce a number between 0 and 1 , and $\mathbb{Q}=q_{1}, \ldots, q_{n}$ is a set of metrics' weights.

Examples of Possible Quality Functions. We discuss two specific quality functions. The goal of the first one, $Q_{1}$, is to minimize the size of the resulting model. Since we assume that there is a large degree of similarity between input models that represent related products of a product line, aiming to reduce the total number of elements in the result leads to a reduction of duplications which, in turn, helps avoid repeating modifications for all variants.

We define our notion of model size using the number of classes, attributes, states and transitions. Specifically, the metrics $v_{1}-v_{4}$ listed in the first four rows of Table 1 measure the overall reduction of the size of the produced model when compared to the inputs. To construct $Q_{1}$, we assign these metrics equal weights, considering them equally important, as specified in the second to last column of Table $1 . Q_{1}$ prefers models that are as compact as possible, e.g., the refactoring in Fig. 1(d, f, h).

Our second goal is to produce refactorings that are the easiest for the user to comprehend. The work of [6]5] makes an argument that an increase in the size of UML models 
Table 1. Quality metrics

\begin{tabular}{|c|c|c|c|c|}
\hline \multirow{2}{*}{\multicolumn{2}{|c|}{ Metric }} & \multirow[t]{2}{*}{ Objective } & \multicolumn{2}{|c|}{ Weight } \\
\hline & & & $Q_{1}$ & $Q_{2}$ \\
\hline \begin{tabular}{l|l}
$v_{1}$ \\
\end{tabular} & $\begin{array}{l}\% \text { of reduction } \\
\text { in \# of classes }\end{array}$ & $\begin{array}{l}\text { Reduce the number of classes. } \\
\text { (out of the total number of classes in input.) }\end{array}$ & 0.25 & 0.125 \\
\hline$v_{2}$ & $\begin{array}{l}\% \text { of reduction } \\
\text { in \# of attributes }\end{array}$ & $\begin{array}{l}\text { Reduce the number of class attributes. } \\
\text { (out of the total number of attributes in input.) }\end{array}$ & 0.25 & 0.125 \\
\hline$v_{3}$ & $\begin{array}{l}\% \text { of reduction } \\
\text { in \# of states }\end{array}$ & $\begin{array}{l}\text { Reduce the number of states. } \\
\text { (out of the total number of states in input.) }\end{array}$ & 0.25 & 0.125 \\
\hline$v_{4}$ & $\begin{array}{l}\% \text { of reduction } \\
\text { in \# of transitions }\end{array}$ & $\begin{array}{l}\text { Reduce the number of transitions. } \\
\text { (out of the total number of transitions in input.) }\end{array}$ & 0.25 & 0.125 \\
\hline$v_{5}$ & $\begin{array}{l}\% \text { of common } \\
\text { attributes }\end{array}$ & $\begin{array}{l}\text { Reduce the percentage of variable class attributes } \\
\text { (out of the total number of the class attributes.) }\end{array}$ & 0.0 & 0.17 \\
\hline$v_{6}$ & $\begin{array}{l}\% \text { of common } \\
\text { states }\end{array}$ & $\begin{array}{l}\text { Reduce an average percentage of variable states } \\
\text { (out of the total number of states in a statechart.) }\end{array}$ & 0.0 & 0.17 \\
\hline$v_{7}$ & $\begin{array}{l}\% \text { of common } \\
\text { transitions }\end{array}$ & $\begin{array}{l}\text { Reduce an average percentage of variable transitions } \\
\text { (out of the total number of transitions in a statechart.) }\end{array}$ & 0.0 & 0.16 \\
\hline
\end{tabular}

(specifically, the number of classes, aggregations, states and transitions) leads to an increase of cognitive complexity. The authors validate this claim using controlled experiments involving human subjects. However, neither these experiments nor our early work [22] considered variability aspects of the annotative product line representations. For example, while they minimize the size of the model, both possible merges of the Dryer and the Beeper classes in Figures $1 \mathrm{~g}$ ) and (h) contain $66 \%$ and $50 \%$ variable states (i.e., states annotated by features), respectively. The merge of the Controller classes in Fig.1 (f) contains $50 \%$ variable states as well.

We believe that the higher is the number of common elements in a merged model, the easier it is to understand. We thus define a second quality function, $Q_{2}$, to combine size minimization with encouraging those refactorings which result in models with a high degree of commonalities: classes with a significant number of common attributes and statecharts with a significant number of common states and transitions. The metrics $v_{5}-v_{7}$ of Table 1 are designed for that purpose. They are calculated by counting the percentage of common sub-elements for a certain element in the model, i.e., those subelements that are annotated by all product line features. To achieve a reasonable degree of merging while discouraging too much variability, $Q_{2}$ gives the combination of four size-based metrics $v_{1}-v_{4}$ and the combination of three variability-based metrics $v_{5}-v_{7}$ equal importance (see the last column of Table 1). This quality function prefers the refactoring in Fig. 11 (c, e).

We use both $Q_{1}$ and $Q_{2}$ to evaluate refactorings of our case-study models in Sec. 5 .

\subsection{Constructing the Desired Refactorings}

Since a quality function captures the criteria that are to be used when performing the merge-refactoring process, it could also be used to guide the process towards the desired result. As stated in Sec. 2, refactorings produced by the merge-in algorithm differ by the way input model elements are matched and merged, which is controlled by the mergein configuration parameters. Modifying these parameters, e.g., increasing weight of 
state name similarities during compare, can result in the refactoring shown in Fig.1(e). Instead, if we give more weight to the structural similarity of states, i.e., their distance to the initial and the final states and, recursively, the similarity of their neighbors [19], we get the result in Fig.1(f). Likewise, lowering the class similarity threshold can result in a refactoring where the Dryer and the Beeper classes are matched and merged together, in addition to merging the Controller classes, as shown in Fig. 1(d)

Obviously, merge-in parameters cannot be decided universally because their values depend on the nature of the refactored product line and the objective of the quality function. It is also unreasonable to assume that the user can set and adjust these parameters manually. Moreover, generating all possible refactorings and evaluating them based on the given quality function is not a feasible approach as it does not scale well.

We thus need a systematic way for identifying those values of merge-in parameters that result in an optimal refactoring w.r.t. the given quality function $Q$. In our work, we propose doing so by treating parameter selection as a classical optimization problem [21], using the chosen quality function as an objective function for an optimization technique. The process (1) uses an optimization heuristic to set values of merge-in parameters, (2) produces the corresponding refactoring, subject to these parameters, (3) evaluates it using $Q$, and repeats until a result of the desired quality is reached (or a certain fixed number of iterations is performed). That is, different refactorings are generated by the merge-in algorithm based on the values of compare weights and similarity thresholds that are set using an optimization algorithm aimed to maximize the value of $Q$. Only the resulting "optimal" refactoring is returned to the user. The overall efficiency of the approach is as good as the chosen optimization algorithm because the latter selects the values of parameters for the next iteration.

\section{Implementation}

In this section, we describe our implementation of the merge-in algorithm, used as a foundation of the merge-refactoring framework, as well as our approach for setting the merge-in parameters. We focus our work on systems represented and implemented with UML models containing class and statechart diagrams - a common choice in automotive, aerospace \& defense, and consumer electronics domains, where such models are often used for full behavioral code generation (e.g., using IBM Rhapsody 1 ).

The core part of the merge-in algorithm is the compare function which receives two UML elements of the same type and returns their similarity degree - a number between 0 and 1. To implement compare, we started with existing comparison algorithms for UML classes [29 13] and statecharts [19]. These algorithms calculate the similarity degree recursively, using formulas that assign empirically defined weights to similarity degrees of appropriately chosen sub-elements.

None of the existing algorithms combined information obtained by analyzing both structural and behavior models together: comparing classes did not take into account information about similarity of their corresponding statecharts. We thus extended class comparison by considering behavior information, obtained by comparing statecharts to each other, and combining it with structural information by giving them equal weights.

${ }^{1}$ http: / /www-01.ibm.com/software/awdtools/rhapsody/ 
We also extended the statechart comparison algorithm proposed in [19] to consider state entry and exit actions, state do activities and actions on transitions, as those were used in the real-life model provided by the industrial partner.

Based on elements' similarity degrees generated by compare, our implementation of match "greedily" selects similar elements that are above a given threshold. Merge further combines elements deemed similar while explicating variabilities. We use the union-merge [26] approach to implement the merge function. It unifies matched elements and copies unmatched elements "as is" to the result. Our merge implementation is an adaptation of static union merge of TReMer +2 , extended to deal with annotations of domain model elements by features, as discussed in Sec. 2. We use IBM Rational Software Architec 3 (RSA) as our modeling environment, allowing us to reuse existing Java-based algorithms. Rhapsody models supplied by our industrial partner were first exported into UML 2.0 XMI format and then imported into RSA.

For adjusting merge-in parameters, we have implemented a version of the local search optimization technique [21] where the space of possible refactorings is explored by changing one parameter value at a time (hill-climbing). After evaluating the resulting refactoring and adjusting this value, we move to the next one, until all values are set. While this algorithm can miss the best result (global maximum) because it does not revisit the decisions that were already made, it is shown to be quite effective in practice for finding a "good" result (local maximum) in an efficient manner. We demonstrate the effect of adjusting the class similarity threshold in Sec.5.

We merge-in the most similar models first: similarity degrees of all inputs - models of individual products or already constructed intermediate product lines - are evaluated, and those with the highest similarity degrees are combined first. Intuitively, merging more similar models first helps decrease the size and the number of variable elements in the result.

During our experiments, we noted that different values of compare weights and similarity thresholds can produce the same refactoring and thus the same quality measurements. Since our goal is to maximize a given quality function, any of the assignments that produce the desired result is appropriate.

\section{Experience}

In this section, we report on our experience applying the quality-based merge-refactorings. Our goal is to validate the feasibility of the approach for UML models in the embedded domain. In particular, we are interested in demonstrating the applicability and effectiveness of the proposed methodology for adjusting merge-in parameters for realistic models containing UML class and statechart diagrams, based on a given quality function. In what follows, we describe our subject product lines and present our results.

Subjects. We applied our refactoring approach to three sets of related products. The first is the Washing Machine example, built by us to mimic a partner's model and to highlight its characteristics. A snippet of this example is presented in Fig. 1 and the full

\footnotetext{
2 http://se.cs.toronto.edu/index.php/TReMer+

${ }^{3}$ http: / /www-01.ibm.com/software/awdtools/swarchitect/
} 
Table 2. Varying Class Similarity Threshold $S_{\text {Class }}$

\begin{tabular}{|c|c|c|c|c|c|c|c|c|c|c|c|c|c|c|c|c|c|}
\hline \multirow[b]{2}{*}{ metrics } & \multicolumn{7}{|c|}{ Washing Machine } & \multicolumn{4}{|c|}{ Microwave Oven } & \multicolumn{6}{|c|}{ CE Product Line } \\
\hline & orig. & 0.7 & 0.75 & 0.78 & 0.8 & 0.85 & 0.9 & orig. & 0.7 & $0.75-0.85$ & 0.9 & $\mid$ orig. & 0.6 & 0.65 & 0.7 & 0.75 & 0.8 \\
\hline \begin{tabular}{l|l}
$v_{1}$ & $\#$ classes
\end{tabular} & $\overline{18}$ & $\overline{6}$ & $\overline{7}$ & 8 & 9 & $\overline{11}$ & $\overline{12}$ & 8 & $\overline{2}$ & $\overline{3}$ & $\overline{5}$ & 45 & $\overline{14}$ & $\overline{15}$ & 17 & $\overline{20}$ & 27 \\
\hline$v_{2}$ \#attributes & 25 & 10 & 12 & 14 & 20 & 25 & 25 & 4 & 1 & 2 & 4 & 104 & 56 & 56 & 75 & 84 & 80 \\
\hline 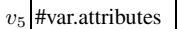 & - & 3 & 4 & 5 & 6 & 4 & 0 & - & 0 & 2 & 4 & - & 43 & 43 & 26 & 32 & 8 \\
\hline$v_{3}$ \#states & 43 & 18 & 20 & 22 & 28 & 38 & 43 & 18 & 7 & 9 & 18 & || 448 & 177 & 151 & 211 & 374 & 412 \\
\hline$v_{6}$ \#var.states & - & 6 & 6 & 5 & 4 & 0 & 0 & - & 1 & 0 & 0 & - & 56 & 64 & 31 & 13 & 4 \\
\hline$v_{4} \mid \#$ transitions & 56 & 28 & 31 & 34 & 40 & 51 & 56 & 44 & 16 & 24 & 44 & 944 & 245 & 260 & 402 & 573 & 640 \\
\hline$v_{7}$ \#var.transitions & - & 19 & 19 & 18 & 12 & 0 & 0 & - & 2 & 4 & 0 & - & 77 & 85 & 31 & 19 & 8 \\
\hline$\overline{\mathrm{Q} 1}$ & - & 0.587 & 0.528 & 0.469 & $\overline{0.333}$ & $\overline{0.148}$ & $\overline{0.083}$ & - & 0.686 & 0.505 & 0.065 & - & 0.646 & $\overline{0.63}$ & $\overline{0.496}$ & $\overline{0.351}$ & $\overline{0.284}$ \\
\hline Q2 & - & 0.565 & 0.560 & 0.572 & 0.533 & 0.523 & 0.541 & - & $\mid 0.797$ & 0.561 & 0.372 & - & 0.640 & 0.65 & 0.678 & 0.601 & 0.623 \\
\hline
\end{tabular}

version is available in [23]. The Washing Machine product line contains three different products, with a high degree of overlap in the set of classes comprising them. Specifically, each product has six classes, out of which three are identical across products (Motor, Faucet and Detergent Supplier), two are similar to each other in all three products (Controller and Washer), and one class in each product carries a unique product-specific functionality (either Dryer, Beeper or Timer). Also, statecharts of similar classes have similar structures.

The second example, Microwave Oven, has been introduced by Gomaa in [9]. It includes four different, although very similar, variants of the timer control class and their corresponding statecharts.

The final example comes from the Consumer Electronics (CE) space, contributed by an industrial partner. Here, we focus on seven behavior-intensive product components which together contain 45 classes, 104 attributes, 448 states and 944 transitions. The number of classes implementing each component ranges between 2 and 14 . The number of statecharts in each component ranges between 1 and 3, with the number of states and transitions for a statechart ranging between 20 and 66 states, and 31 and 81 transitions, respectively. Of the seven components, three have a similar structure and a similar set of elements; another pair of components also contains elements that are similar to each other (but less similar to the components of the first cluster), and the remaining two components are not similar to the rest.

Space limitations and verbosity of UML models do not allow us to include pictorial illustrations of the examples. Thus, we limit the presentation to the statistical data about the case studies. The complete models for the first two examples are available in [23]. Since we cannot share details of the CE model, we built our first example, the Washing Machine, to be similar.

Results. To evaluate the effectiveness of our quality-based merge-refactoring approach, we analyzed different refactorings produced by varying compare weights and similarity thresholds, and evaluated them using quality functions $Q_{1}$ and $Q_{2}$ introduced in Sec.3. As a starting point, we used empirically determined weights specified in [29 13 19]. We updated the weights to combine structural and behavior information when comparing classes and to take into account additional statechart elements, as described in Sec. 4 . For the similarity thresholds, we started with the assumption that elements with the similarity degree lower than 0.5 are significantly different and should not be combined. For statecharts, we refined these estimates using the thresholds empirically determined in [19]. The values of weights and thresholds that we used are summarized in [23]. 
For illustration purposes, in this section we vary the class similarity threshold between 0.4 and 1 , iteratively incrementing its value by 0.01 , and evaluate the produced results using our quality functions. Table 2 presents the total number of elements as well as the number of variable elements of each type in the resulting refactoring. To save space, we show only distinct refactorings, omitting those that are equivalent to the presented ones. For example, in the Washing Machine case, all refactorings produced with class similarity thresholds between 0.4 and 0.7 are identical, and we only show the latest. In addition, the orig column reports the total number of input elements for each of the case studies. It is used to compare the result of the refactoring to the original models and to normalize the collected metrics during quality computation. A full description of the refactored product line models that were produced for each step of the first two case-studies is available in [23].

The results demonstrate that increasing the value of the class similarity threshold results in decreasing the value of $Q_{1}$ in all case studies because this function prefers refactorings that are as compact as possible: as the class similarity threshold increases, fewer classes are matched and merged, and the number of elements in the result grows. $Q_{2}$, however, does not exemplify such linear behavior because it balances the reduction in size with the goal of merging only those elements that are indeed similar. For example, when refactoring the Washing Machine, the result preferred by $Q_{1}$ is obtained by setting the class similarity threshold to 0.7 or lower, which causes merging of as many classes as possible, including those that are dissimilar (e.g., the one in Fig. 1(d)). This produces state machines with a large percentage of variable states and transitions. $Q_{2}$ prefers the solution produced when the similarity threshold is set to 0.78 , which merges only elements with a high degree of commonality (e.g., see Fig. 11 c)). When the class similarity threshold is high (0.9), only identical classes got merged. A large number of classes, states and transition in the resulting model is captured by a low calculated value for both $Q_{1}$ and $Q_{2}$, since both of them are designed to minimize the size of the result.

For the Microwave Oven example, both $Q_{1}$ and $Q_{2}$ prefer the solution found when the class similarity threshold is set to 0.7 or lower (see Table 2). Since all four variants of the timer control class in this example are very similar, these classes are all merged together in the resulting refactoring. The percentage of variable states and transitions in this solution remains small, and the overall reduction in their total number is significant.

Recall that our third example had two clusters of similar components (and two other components, different from the rest). The refactoring that identifies and merges components in these clusters is produced when the class similarity threshold is set to 0.7. This refactoring also maximizes the value of $Q_{2}$. Similarly to the Washing Machine case, lower threshold values produce more merges resulting in a high number of variable attributes, states and transitions (and thus, lower values of $Q_{2}$ ), while higher thresholds result in a large number of elements in the resulting model (and thus, lower values of both $Q_{1}$ and $Q_{2}$ ).

In summary, we found that in all of the above cases, quality functions were able to distinguish between different refactorings as desired and thus were appropriate to help "drive" the refactoring process towards the preferable result. Our third case study also showed that differences in the computed quality values became more pronounced as models got bigger. Furthermore, the refactorings that were produced in our examples 
under the strategy that maximizes the value of $Q_{2}$ were identical to those constructed manually by a domain expert. This encouraging result makes us believe that our qualitybased merge-refactorings approach is effective for the creation of annotative product line representations from a set of existing systems.

Threats to Validity. Threats to external validity are most significant for our work. These arise when the observed results cannot generalize to other case studies. Because we used a limited number of subjects and quality functions, our results might not generalize without an appropriate tuning. However, we attempted to mitigate this threat by using a real-life case study of considerable size as one of our examples. Thus, even though preliminary, our results show that the approach, perhaps with some additional tuning, is effective for finding good refactorings of large-scale systems.

In addition, we limit the scope of our work to physical systems in the embedded domain, where number of product variants usually does not exceed tens. The approach might not scale well to other domains, where hundreds of product variants are possible. However, we believe that the scalability issue mostly relates to the annotative product line representation itself, rather than to our attempt to distinguish between different representations.

\section{Discussion and Related Work}

Product Line Refactoring Approaches. Several existing approaches aim at building product lines out of legacy artifacts, e.g., [8]. These approaches mainly provide guidelines and methodologies for identifying features and their related implementation components rather than build tool-supported analysis mechanisms. Some works also report on successful experience in manual re-engineering of legacy systems into featureoriented product lines, e.g., [14].

Koschke et al. [15] present an automated technique for comparing software variants at the architectural level and reconstructing the system's static architectural view which describes system components, interfaces and dependencies, as well their grouping into subsystems. Ryssel et al. [25] introduce an automatic approach to re-organize Matlab model variants into annotative representations while identifying variation points and their dependencies. Yoshimura et al. [30] detect variability in a software product line from its change history. None of the above approaches, however, takes into account quality attributes of the constructed results nor attempt to distinguish between the different refactorings based on the refactoring goal.

Product Line Quality. Oliveira et al. [20] propose a metric suite to support evaluation of product line architectures based on McCabe's cyclomatic complexity of their core components, which is computed using the control flow graph of the program and measures the number of linearly independent paths through a program's source code. Her et al. [10] suggest a metric to measure reusability of product line core assets based on their ability to provide functionality to many products of the same SPL, the number of SPL variation points that are realized by an asset, the number of replaceable components in a core asset and more. Hoek et al. [28] describe metrics for measuring service utilization of SPL components based on the percentage of provided and required services of a component. While these works allow measuring reusability, extensibility and 
implementation-level complexity of product line core assets, they do not discuss the structural complexity of annotative SPL models nor allow comparing different annotative product line models and distinguishing between them based on their representation properties. Trendowicz and Punter [27] investigate to which extend existing quality modeling approaches facilitate high quality product lines and define requirements for an appropriate quality model. They propose a goal-oriented method for modeling quality during the SPL development lifecycle, but do not propose any concrete metrics.

Numerous works, e.g., [65], propose software metrics for evaluating quality of UML models. While we base our approach on some of these works, they are not designed for UML models that represent software product lines and do not take variability aspects into account.

Finally, some works discuss characteristics of feature implementations in code, such as feature cohesion and coupling [1] or granularity, frequency and structure of preprocessor annotations [12 16]. However, these works are not easily generalizable to address the issue of structural complexity of models.

\section{Conclusions and Future Work}

Understanding and refactoring existing legacy systems can promote product line adoption by industrial organizations which have made a significant investment in building and maintaining these systems, and are not ready to abandon them for "starting from scratch". Since these systems are usually very large, automation becomes a necessity.

In this paper, we focused on integrating distinct products specified in UML into an annotative product line representation. We argued that multiple syntactically different yet semantically equivalent representations of the same product line model are possible, and the goal of the refactoring induces which one is preferable. We suggested an approach for guiding the refactoring process towards a result that fits best the user's intention, as captured by a syntactic quality function. We implemented a refactoring algorithm based on model merging and used it as the foundation of our merge-refactoring framework. We evaluated the proposed quality-based merge-refactoring approach on a set of case-studies, including a large-scale example contributed by an industrial partner. We believe that our work promotes automation of product line refactoring and reasoning about refactoring alternatives.

For future work, we are interested in enhancing our understanding of product line quality considerations which can help with assessing different product line model representations, produced either automatically or manually. The quality functions can be extended to consider additional quality attributes, allow the user to set and/or interactively choose different quality goals for different regions within the model, incorporate user feedback and more. Performing user studies for evaluating quality of annotative product line models can also be a subject of future work.

In addition, we are interested in exploring more sophisticated refactoring techniques that are able to detect fine-grained features in the combined products. This will allow creating new products in the product line by "mixing" features from different original products, e.g., the dryer and the beeper features from the models in Fig. 1 We also plan to further improve our match algorithms by allowing the user to affect results of this 
function, e.g., by setting negative matches. Exploring the use of more advanced optimization techniques, such as cross-entropy for adjusting compare and match parameters is also a subject for possible future work.

Acknowledgments. We thank Andrei Kirshin (IBM Research) for useful discussions and the anonymous reviewers for their insightful comments.

\section{References}

1. Apel, S., Beyer, D.: Feature Cohesion in Software Product Lines: an Exploratory Study. In: Proc. of ICSE 2011, pp. 421-430 (2011)

2. Beuche, D.: Transforming Legacy Systems into Software Product Lines. In: Bosch, J., Lee, J. (eds.) SPLC 2010. LNCS, vol. 6287, pp. 509-510. Springer, Heidelberg (2010)

3. Boucher, Q., Classen, A., Heymans, P., Bourdoux, A., Demonceau, L.: Tag and Prune: a Pragmatic Approach to Software Product Line Implementation. In: Proc. of ASE 2010, pp. 333-336 (2010)

4. Clements, P.C., Northrop, L.: Software Product Lines: Practices and Patterns (2001)

5. Cruz-Lemus, J.A., Genero, M., Piattini, M.: Using Controlled Experiments for Validating UML Statechart Diagrams Measures. In: Cuadrado-Gallego, J.J., Braungarten, R., Dumke, R.R., Abran, A. (eds.) IWSM-Mensura 2007. LNCS, vol. 4895, pp. 129-138. Springer, Heidelberg (2008)

6. Esperanza Manso, M., Cruz-Lemus, J.A., Genero, M., Piattini, M.: Empirical Validation of Measures for UML Class Diagrams: A Meta-Analysis Study. In: Chaudron, M.R.V. (ed.) MODELS 2008 Workshops. LNCS, vol. 5421, pp. 303-313. Springer, Heidelberg (2009)

7. Faust, D., Verhoef, C.: Software Product Line Migration and Deployment. Journal of Software Practice and Experiences 30(10), 933-955 (2003)

8. Ferber, S., Haag, J., Savolainen, J.: Feature Interaction and Dependencies: Modeling Features for Reengineering a Legacy Product Line. In: Chastek, G.J. (ed.) SPLC 2002. LNCS, vol. 2379, pp. 235-256. Springer, Heidelberg (2002)

9. Gomaa, H.: Designing Software Product Lines with UML: From Use Cases to Pattern-Based Software Architectures. Addison Wesley (2004)

10. Her, J.S., Kim, J.H., Oh, S.H., Rhew, S.Y., Kim, S.D.: A Framework for Evaluating Reusability of Core Asset in Product Line Engineering. Information and Software Technology 49(7), 740-760 (2007)

11. Kästner, C., Apel, S.: Integrating Compositional and Annotative Approaches for Product Line Engineering. In: Proc. of GPCE Workshops (McGPLE 2008), pp. 35-40 (2008)

12. Kästner, C., Apel, S., Kuhlemann, M.: Granularity in Software Product Lines. In: Proc. of ICSE 2008, pp. 311-320 (2008)

13. Kelter, U., Wehren, J., Niere, J.: A Generic Difference Algorithm for UML Models. In: Software Engineering. LNI, vol. 64, pp. 105-116 (2005)

14. Kolb, R., Muthig, D., Patzke, T., Yamauchi, K.: Refactoring a Legacy Component for Reuse in a Software Product Line: a Case Study: Practice Articles. J. of Software Maintenance and Evolution 18(2), 109-132 (2006)

15. Koschke, R., Frenzel, P., Breu, A.P., Angstmann, K.: Extending the Reflexion Method for Consolidating Software Variants into Product Lines. Software Quality Control 17(4), 331-366 (2009)

16. Liebig, J., Apel, S., Lengauer, C., Kästner, C., Schulze, M.: An Analysis of the Variability in Forty Preprocessor-Based Software Product Lines. In: Proc. of ICSE 2010, pp. 105-114 (2010) 
17. McQuillan, J.A., Power, J.F.: On the Application of Software Metrics to UML Models. In: Kühne, T. (ed.) MoDELS 2006 Workshops. LNCS, vol. 4364, pp. 217-226. Springer, Heidelberg (2007)

18. Mende, T., Koschke, R., Beckwermert, F.: An Evaluation of Code Similarity Identification for the Grow-and-Prune Model. J. of Software Maintenance and Evolution 21(2), 143-169 (2009)

19. Nejati, S., Sabetzadeh, M., Chechik, M., Easterbrook, S., Zave, P.: Matching and Merging of Statecharts Specifications. In: Proc. of ICSE 2007, pp. 54-64 (2007)

20. Oliveira Junior, E., Maldonado, J., Gimenes, I.: Empirical Validation of Complexity and Extensibility Metrics for Software Product Line Architectures. In: Proc. of SBCARS 2010, pp. 31-40 (2010)

21. Pardalos, P., Resende, M.: Handbook of Applied Optimization (2002)

22. Rubin, J., Chechik, M.: From Products to Product Lines Using Model Matching and Refactoring. In: Proc. of SPLC Wrksp, MAPLE 2010, pp. 155-162 (2010)

23. Rubin, J., Chechik, M.: (2012), http: //www.cs.toronto.edu/ mjulia/PLRefactoring/

24. Rubin, J., Chechik, M.: Combining Related Products into Product Lines. In: de Lara, J., Zisman, A. (eds.) FASE 2012. LNCS, vol. 7212, pp. 285-300. Springer, Heidelberg (2012)

25. Ryssel, U., Ploennigs, J., Kabitzsch, K.: Automatic Variation-Point Identification in Function-Block-Based Models. In: Proc. of GPCE 2010, pp. 23-32 (2010)

26. Sabetzadeh, M., Easterbrook, S.: View Merging in the Presence of Incompleteness and Inconsistency. Requirement Engineering 11, 174-193 (2006)

27. Trendowicz, A., Punter, T.: Quality Modeling for Software Product Lines. In: Proc. of ECOOP Workshops, QAOOSE 2003 (2003)

28. van der Hoek, A., Dincel, E., Medvidovic, N.: Using Service Utilization Metrics to Assess the Structure of Product Line Architectures. In: Proc. METRICS 2003, pp. 298-308 (2003)

29. Xing, Z., Stroulia, E.: UMLDiff: an Algorithm for Object-Oriented Design Differencing. In: Proc. of ASE 2005, pp. 54-65 (2005)

30. Yoshimura, K., Narisawa, F., Hashimoto, K., Kikuno, T.: FAVE: Factor Analysis Based Approach for Detecting Product Line Variability from Change History. In: Proc. of MSR 2008, pp. 11-18 (2008) 